\title{
Prevalence and possible causes of hypouricemia at a tertiary care hospital
}

\author{
Chang-Nam Son ${ }^{1}$, Ji-Min Kim ${ }^{1}$, Sang-Hyon Kim ${ }^{1}$, Soo-Kyung $\mathrm{Cho}^{2}$, Chan-Bum Choi ${ }^{2}$, \\ Yoon-Kyoung Sung ${ }^{2}$, Tae-Hwan Kim², Sang-Cheol Bae ${ }^{2}$, Dae-Hyun $\mathrm{Yoo}^{2}$, and Jae-Bum Jun ${ }^{2}$
}

${ }^{1}$ Department of Internal Medicine, Keimyung University Dongsan Medical Center, Daegu; ${ }^{2}$ Department of Rheumatology, Hospital for Rheumatic Diseases, Hanyang University College of Medicine, Seoul, Korea

Received: April 30, 2015

Revised : June 4, 2015

Accepted: June 25, 2015

\section{Correspondence to}

Jae-Bum Jun, M.D.

Department of Rheumatology, Hospital for Rheumatic Diseases, Hanyang University College of Medicine, 222-1 Wangsimni-ro, Seongdong-gu, Seoul 04763, Korea Tel: +82-2-2290-9216

Fax: +82-2-2298-8231

E-mail:junjb@hanyang.ac.kr
Background/Aims: We aimed to investigate the prevalence and possible causes of hypouricemia in the Korean population and to compare our findings with published results of other populations.

Methods: We examined the serum uric acid levels of 30,757 subjects who had their uric acid values measured at least once during a 1-year period. All individuals with hypouricemia (serum uric acid $<2.0 \mathrm{mg} / \mathrm{dL}, \mathrm{n}=424$ ) were reviewed with respect to medical drug history and concomitant diseases previously identified as being associated with hypouricemia.

Results: The prevalence of hypouricemia was $4.14 \%(299 / 7,223)$ among inpatients and $0.53 \%(125 / 23,534)$ among outpatients, for an overall prevalence of $1.39 \%$ $(424 / 30,757)$. Possible causes associated with hypouricemia were found to be solid or hematologic malignancies $(n=86)$, diabetes mellitus $(n=56)$, and therapeutic drugs $(n=29)$. The medications were allopurinol $(n=11)$, angiotensin II receptor blockers $(n=10)$, salicylates $(n=6)$, febuxostat $(n=1)$, and warfarin $(n=1)$. In the remaining 226 individuals, the cause of hypouricemia was not identified.

Conclusions: Hypouricemia is relatively common in the Korean population compared to those of other countries. The possible causes associated with hypouricemia are related to underlying diseases and medications.

Keywords: Hypouricemia; Uric acid

\section{INTRODUCTION}

Hypouricemia is generally defined as a serum uric acid (SUA) concentration of less than $2.0 \mathrm{mg} / \mathrm{dL}$ [1]. It has no recognizable symptoms that require treatment. It is characterized by increased uric acid clearance or decreased uric acid production $[1,2]$. While hyperuricemia and gout are significant health concerns and have been the subject of a large number of studies [3,4], less attention has been paid to hypouricemia, even though it is clinically important. Nonetheless, interest in hypouricemia has begun to increase, due to a better understanding of the role of uric acid transporters. The human urate transporter 1 (URAT1) and human glucose transporterlike protein 9 (GLUT9) are two representative renal urate transporters [2,5-11]. A genetic defect in these two urate transporters is responsible for idiopathic hypouricemia.

The reported prevalence of hypouricemia has varied in previous studies, depending on the type of study subject and the definition of hypouricemia [5,12-20]. Some studies have reported that it was between $0.15 \%$ and $3.30 \%$ in the general population and in outpatients and between $1.24 \%$ and $2.54 \%$ in hospitalized patients. A higher prevalence of hypouricemia has been found among inpatients than among outpatients. In addition to a genetic defect of the renal urate transporter, 
enhanced uric acid excretion due to medical diseases or medications can also result in hypouricemia [1].

The prevalence of hypouricemia has not been investigated previously in Korea and few studies have focused on the factors affecting hypouricemia in the Korean population. Therefore, we aimed to investigate the prevalence and possible causes of hypouricemia in the Korean population and to compare our findings with those reported for other populations.

\section{METHODS}

\section{Population sample}

A total of 48,613 SUA values were obtained from patients who visited a tertiary care hospital for medical treatment or a checkup during the period January to December 2012. In these 12 months 30,757 patients received at least one SUA test, and 7,223 patients had the test while they were hospitalized. Although 24,970 subjects visited the outpatient clinic, the number of outpatients was calculated as 23,534 because those who had already had an SUA test while hospitalized during that period were excluded if they had the test again as outpatients. If a patient had multiple SUA values during the test period, if at least one of those values was less than $2.0 \mathrm{mg} / \mathrm{dL}$, he/she was classified in the hypouricemia patient group. The total number of hypouricemia patients was 424 .

\section{SUA levels of the study subjects}

The medical information team at the hospital used a computer program to record the patients' SUA levels. Hypouricemia was identified as cases in which the SUA level was less than $2.0 \mathrm{mg} / \mathrm{dL}[1,19]$. An average of each patient's SUA values was taken as a representative SUA value for each patient. However, in the hypouricemia group, if a patient only had one SUA value that was less than $2.0 \mathrm{mg} / \mathrm{dL}$, that measurement was taken as his/her representative value. Moreover, in cases where there were more than two values of SUA less than $2.0 \mathrm{mg} / \mathrm{dL}$, the values exceeding $2.0 \mathrm{mg} / \mathrm{dL}$ were excluded, and only those less than $2.0 \mathrm{mg} / \mathrm{dL}$ were used for calculating the average to obtain the representative value.

\section{Review of possible causes of hypouricemia}

The hospitalization records, outpatient medication re- cords, and underlying diseases of the subjects with hypouricemia were assessed for the 3 months before the occurrence of hypouricemia. If there were recorded medications from another hospital, they were included as etiologies, and the most probable etiologies were selected.

\section{Statistical analysis}

Continuous variables, such as the representative SUA levels, are expressed as the mean \pm standard deviation. Categorical variables, such as the cause of hypouricemia, are shown as frequencies. Student $t$ test was used to examine differences in the SUA levels of the subjects with hypouricemia. A p value of less than 0.05 was considered statistically significant. All statistical analyses were performed using SPSS version 20.0 (IBM Co., Armonk, NY, USA).

\section{Ethics statement}

This study was approved by the Institutional Review Board of Hanyang University Hospital. Informed consent from the patients was not required because we examined the data retrospectively from medical records and de-identified it after collection to ensure patient confidentiality.

\section{RESULTS}

\section{Characteristics of the study subjects}

Among the 30,757 subjects, $57.6 \%$ were female $(n=17,725)$ and $42.4 \%$ were male $(n=13,032)$. The total mean age was $50.8 \pm 15.7$ years; the mean age of the female subjects was $51.5 \pm 15.5$ years and the mean age of the male subjects was $49.9 \pm 16.0$ years. Mean SUA level was $5.16 \pm 1.62 \mathrm{mg} /$ $\mathrm{dL}$; the mean SUA level of the male subjects $(6.05 \pm 1.60$ $\mathrm{mg} / \mathrm{dL}$ ) was higher than that of the female subjects ( 4.50 $\pm 1.27 \mathrm{mg} / \mathrm{dL}, p<0.05)$.

\section{Prevalence of hypouricemia}

The prevalence of hypouricemia among all of the subjects was $1.39 \%(424 / 30,757)$ (Fig. 1). Among the 424 subjects with hypouricemia, $68.4 \%(n=290)$ were female and $31.6 \%(n=134)$ were male. The prevalence of hypouricemia was $4.14 \%(299 / 7,223)$ among inpatients and $0.53 \%$ $(125 / 23,534)$ among outpatients, and $1.03 \%(134 / 13,032)$ in 


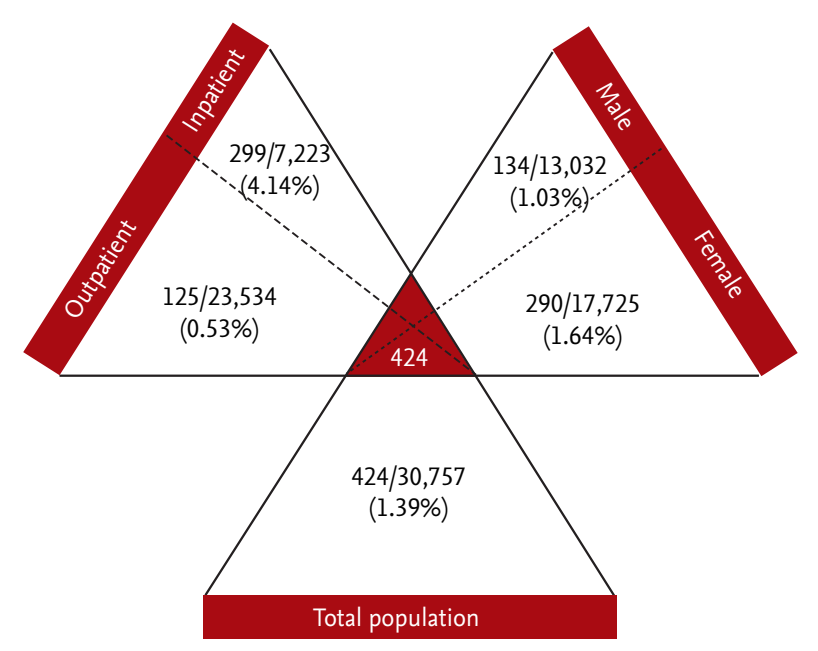

Figure 1. The prevalence of hypouricemia in total population, male, female, inpatient, and outpatient.

male patients and $1.64 \%(290 / 17,725)$ in female patients.

\section{Possible causes associated with hypouricemia}

Table 1 presents the possible causes of hypouricemia and their rates among the patients enrolled in the study. The possible causes were identified as solid or hematologic malignancies $(n=86)$, diabetes mellitus $(n=56)$, and therapeutic drugs $(n=29)$. The medications were allopurinol $(\mathrm{n}=11)$, angiotensin II receptor blockers $(\mathrm{n}=$ 10), salicylates $(n=6)$, febuxostat $(n=1)$, and warfarin $(n=$ 1). In the remaining 226 individuals, the cause of hypouricemia was not identified.

\section{DISCUSSION}

In the present study, the prevalence of hypouricemia among Koreans was relatively high, and it was detected in inpatients as well as outpatients and healthy groups [12-19]. As in other studies, the prevalence of hypouricemia was higher in inpatients than in outpatients or healthy people $[15,17-19]$. According to data collected in studies that used a similar definition of hypouricemia, the total prevalence rate of hypouricemia among westerners varied from $0.61 \%$ to $0.97 \%[13,16]$. In Japan, the prevalence rate for outpatients and healthy individuals ranged between $0.15 \%$ and $0.34 \%$, whereas that of inpatients was $2.54 \%[15,17]$. In our study, the total prevalence
Table 1. Possible causes associated with hypouricemia in Korean population

\begin{tabular}{lc}
\hline Possible causes & No. (\%) \\
\hline Neoplasia & $86(43.4)$ \\
Solid & $79(39.9)$ \\
\hline Hematologic & $7(3.5)$ \\
Diabetes mellitus & $56(28.3)$ \\
\hline Therapeutic drugs & $29(14.6)$ \\
\hline Allopurinol & $11(5.6)$ \\
\hline Angiotensin II receptor blockers & $10(5.1)$ \\
\hline Salicylates & $6(3.0)$ \\
Febuxostat & $1(0.5)$ \\
Warfarin & $1(0.5)$ \\
Intracranial disease & $18(9.1)$ \\
Obstructive jaundice & $7(3.5)$ \\
\hline Renal tubular acidosis & $1(0.5)$ \\
\hline Ulcerative colitis & $1(0.5)$ \\
\hline Total & 198 \\
\hline
\end{tabular}

rate was $1.39 \%$, which is higher than that of westerners; the prevalence rate of outpatients and checkup patients was $0.53 \%$, and that of inpatients was $4.14 \%$. The prevalence rates were higher compared to those reported for Japanese patients. The prevalence rates are summarized in Table 2. In a previous study, the prevalence of hypouricemia among 909 healthy Koreans was 3.3\% [20]. However, the criterion for hypouricemia in that study was less than $3.0 \mathrm{mg} / \mathrm{dL}$, so the results cannot be compared with those of our study. Applying the definition of an SUA value of less than $3.0 \mathrm{mg} / \mathrm{dL}$ in our data analysis, the prevalence rate was $7.66 \%(2,355 / 30,757)$, and even when comparing only the outpatients and check-up patients, it was as high as 5.23\% (1,306/24,971). The prevalence was higher among women than among men $[12,14,17,20]$. This finding is natural, because the number of medications or diseases that might trigger hypouricemia in inpatients is greater [18], and it is well known that the SUAs of premenopausal women are lower than men's [21]. The cause of the higher prevalence of hypouricemia among Koreans than among other ethnicities is not clear. Although the exact reason is unknown, it should be associated with genetic differences, body weight, and dietary habits. Nevertheless, given the prevalence rate, more research and attention must be 


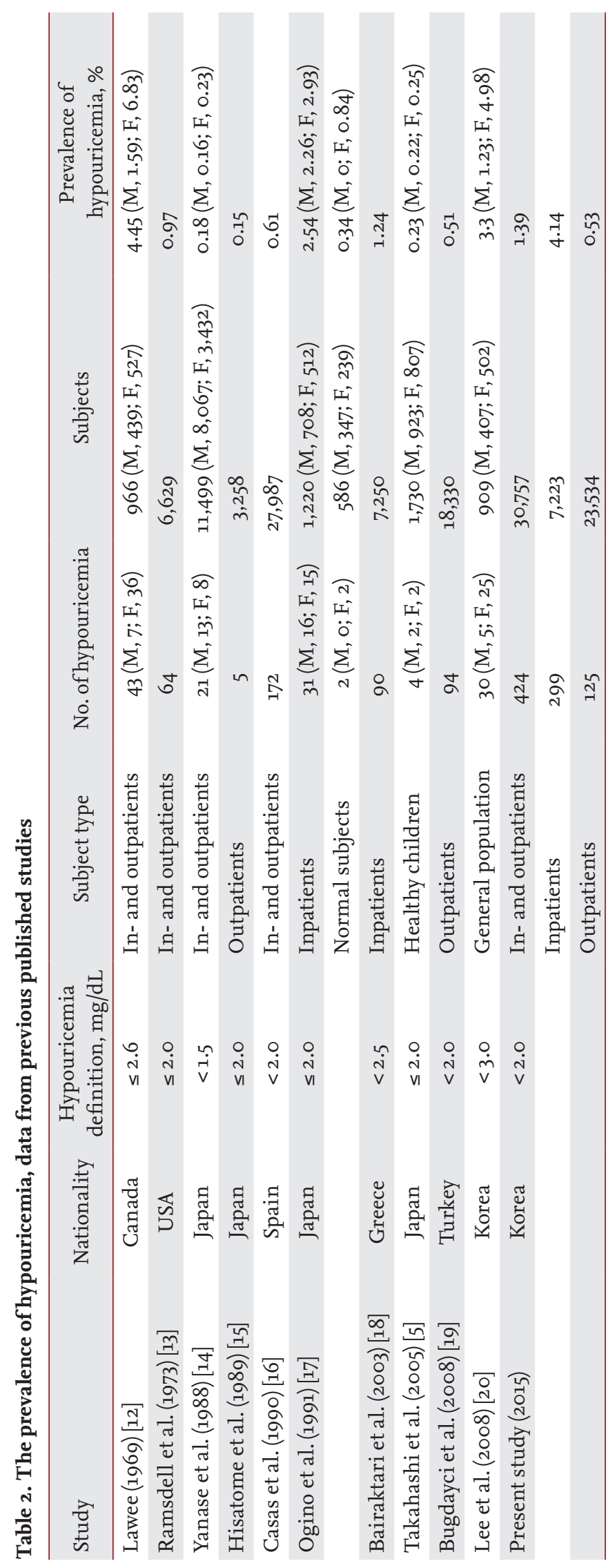

applied to hypouricemia, considering the great deal of interest in hyperuricemia.

Malignancy ranked first as a possible etiology of hypouricemia [22,23], followed by diabetes mellitus and concomitant medication $[24,25]$. The rate of obstructive jaundice or liver disease as etiologies was relatively low [19]. These results are consistent with previous research outcomes. In order to differentiate hypouricemia, a fractional excretion of uric acid (FEUA) is necessary to evaluate renal tubular defects [1,2]. When FEUA is over $10 \%$, it is considered that the subject has pathologic uricosuria. When FEUA decreases, it means that uric acid production has declined, while the reason FEUA increases is a decrease in reabsorption or an increase in the renal excretion of uric acid. When hypouricemia is detected in a hospital, efforts should be made to find the cause. Whether there is another disease causing it should also be checked; hypouricemia can be a tool for finding underlying disease.

Hypouricemia patients are known to have no symptoms, so any symptoms that appear are those of a causative disease of hypouricemia [1]. According to reports on idiopathic renal hypouricemia (iRHUC), patients exhibits exercise-induced acute renal failure, urolithiasis, and hematuria along with fatigue, nausea, vomiting, and diffuse abdominal discomfort as causative diseases of hypouricemia $[1,2,10,26]$. Uric acid is a strong antioxidant, and anaerobic exercise increases oxygen-free radicals, which then trigger kidney vasoconstriction and reduce the glomerular filtration rate [27]. In Japan, it is argued that iRHUC can be found by testing SUA before the initiation of a physical education class [26]. In order to diagnose iRHUC, hypouricemia and increased FEUA should first be checked [2]. There are two different types of genetic defects of the renal urate transporter $[10,28]$. The first type is a defect of the SLC22A12 gene encoding in URAT1 [29], and the second type is a defect of the SLC2A9 gene encoding GLUT9 [30]. Among the 424 subjects with hypouricemia in our study, 226 cases were of an unknown cause. Unfortunately, only a little FEUA was measured during the measurement of SUA, and therefore, the possibility of iRHUC was not verified. However, it was possible in a considerable number of cases. Therefore, when the cause of hypouricemia is unclear, efforts to find iRHUC through FEUA and genetic testing should be made. 
The present study had some limitations. As mentioned earlier, an investigation of test results in a single medical institution was conducted. While a previous study of Koreans involved 30 hypouricemia patients, this study is meaningful because we analyzed the prevalence and causes of this disease with a large number of subjects. Second, there might be information missing, such as height, weight, and renal function, because this study only investigated medical records of medication and comorbidities to determine the causes of hypouricemia. Interviews with patients, FEUA, and genetic testing will all lead to a more precise analysis. Lastly, comparisons with other ethnicities with respect to the prevalence of hypouricemia and its causes require caution in interpretation, because the results might differ according to the prevalence of underlying diseases and the frequency of using medication.

In conclusion, hypouricemia is relatively common in the Korean population. The possible causes associated with hypouricemia are related to underlying diseases and medications. In addition, idiopathic hypouricemia seems not to be rare.

\section{KEY MESSAGE}

1. Hypouricemia is relatively common in the Korean population compared to those of other countries.

2. The possible causes associated with hypouricemia are related to underlying diseases and medications.

\section{Conflict of interest}

No potential conflict of interest relevant to this article was reported.

\section{Acknowledgments}

This work was supported by the research fund of Hanyang University (HY-2012-MC).

\section{REFERENCES}

1. Esparza Martin N, Garcia Nieto V. Hypouricemia and tubular transport of uric acid. Nefrologia 2011;31:44-50.
2. Sebesta I, Stiburkova B, Bartl J, et al. Diagnostic tests for primary renal hypouricemia. Nucleosides Nucleotides Nucleic Acids 2011;30:1112-1116.

3. Kim HS. Carpal tunnel syndrome caused by tophaceous gout. Korean J Intern Med 2014;29:544-545.

4. Park JW, Ko DJ, Yoo JJ, et al. Clinical factors and treatment outcomes associated with failure in the detection of urate crystal in patients with acute gouty arthritis. Korean J Intern Med 2014;29:361-369.

5. Takahashi T, Tsuchida S, Oyamada T, et al. Recurrent URATı gene mutations and prevalence of renal hypouricemia in Japanese. Pediatr Nephrol 2005;20:576-578.

6. Cheong HI, Kang JH, Lee JH, et al. Mutational analysis of idiopathic renal hypouricemia in Korea. Pediatr Nephrol 2005;20:886-890.

7. Ichida K, Hosoyamada M, Hisatome I, et al. Clinical and molecular analysis of patients with renal hypouricemia in Japan-influence of URATr gene on urinary urate excretion. J Am Soc Nephrol 2004;15:164-173.

8. Iwai N, Mino Y, Hosoyamada M, Tago N, Kokubo Y, Endou H. A high prevalence of renal hypouricemia caused by inactive SLC22A12 in Japanese. Kidney Int 2004;66:935944 .

9. Hamajima N, Naito M, Hishida A, Okada R, Asai Y, Wakai K. Serum uric acid distribution according to SLC22A12 W258X genotype in a cross-sectional study of a general Japanese population. BMC Med Genet 2011;12:33.

10. Shen H, Feng C, Jin X, et al. Recurrent exercise-induced acute kidney injury by idiopathic renal hypouricemia with a novel mutation in the SLC2A9 gene and literature review. BMC Pediatr 2014;14:73.

11. Jeannin G, Chiarelli N, Gaggiotti M, et al. Recurrent exercise-induced acute renal failure in a young Pakistani man with severe renal hypouricemia and $\mathrm{SLC}_{2} \mathrm{~A}_{9}$ compound heterozygosity. BMC Med Genet 2014;15:3.

12. Lawee D. Uric acid: the clinical application of 1000 unsolicited determinations. Can Med Assoc J 1969;100:838-841.

13. Ramsdell CM, Kelley WN. The clinical significance of hypouricemia. Ann Intern Med 1973;78:239-242.

14. Yanase M, Nakahama H, Mikami H, Fukuhara Y, Orita Y, Yoshikawa H. Prevalence of hypouricemia in apparently normal population. Nephron 1988;48:80.

15. Hisatome I, Ogino K, Kotake H, et al. Cause of persistent hypouricemia in outpatients. Nephron 1989;51:13-16.

16. Casas E, Serrano C, Daimiel E, Michan A, Mateos F, Garcia Puig J. Prevalence, physiopathology and processes as- 
sociated with hypouricemia in a hospitalized population: analysis of 27,987 analytic determinations. Rev Clin Esp 1990;186:211-215.

17. Ogino K, Hisatome I, Saitoh M, et al. Clinical significance of hypouricemia in hospitalized patients. J Med 1991;22:76-82.

18. Bairaktari ET, Kakafika AI, Pritsivelis N, et al. Hypouricemia in individuals admitted to an inpatient hospital-based facility. Am J Kidney Dis 2003;41:1225-1232.

19. Bugdayci G, Balaban Y, Sahin O. Causes of hypouricemia among outpatients. Lab Med 2008;39:550-552.

20. Lee JH, Choi HJ, Lee BH, et al. Prevalence of hypouricaemia and SLC22A12 mutations in healthy Korean subjects. Nephrology (Carlton) 2008;13:661-666.

21. Johnson RJ, Rideout BA. Uric acid and diet: insights into the epidemic of cardiovascular disease. N Engl J Med 2004;350:1071-1073.

22. Lesmes A, Diaz-Curiel M, Castrillo JM. Tumoural hypouricemia. Adv Exp Med Biol 1980;122A:145-148.

23. Kelly WN. Hypouricemia. Arthritis Rheum 1975;18(6 Suppl):731-737.

24. Shichiri M, Iwamoto H, Shiigai T. Diabetic renal hypouri- cemia. Arch Intern Med 1987;147:225-228.

25. Bo S, Cavallo-Perin P, Gentile L, Repetti E, Pagano G. Hypouricemia and hyperuricemia in type 2 diabetes: two different phenotypes. Eur J Clin Invest 2001;31:318-321.

26. Nakamura A, Niimi R, Yanagawa Y. Renal hypouricemia in school-aged children: screening of serum uric acid level before physical training. Pediatr Nephrol 2006;21:18981900.

27. Ames BN, Cathcart R, Schwiers E, Hochstein P. Uric acid provides an antioxidant defense in humans against oxidant- and radical-caused aging and cancer: a hypothesis. Proc Natl Acad Sci U S A 1981;78:6858-6862.

28. Sebesta I, Stiburkova B. Purine disorders with hypouricemia. Prilozi 2014;35:87-92.

29. Stiburkova B, Sebesta I, Ichida K, et al. Novel allelic variants and evidence for a prevalent mutation in URAT1 causing renal hypouricemia: biochemical, genetics and functional analysis. Eur J Hum Genet 2013;21:1067-1073.

30. Vitart V, Rudan I, Hayward C, et al. SLC2A9 is a newly identified urate transporter influencing serum urate concentration, urate excretion and gout. Nat Genet 2008;40:437-442. 